\title{
Sixteen-month-olds comprehend unanchored absent reference
}

\author{
Elena Luchkina (elena.luchkina@northwestern.edu) \\ Department of Psychology, Northwestern University, Evanston IL, 60208 \\ Fei Xu (fei xu@berkeley.edu) \\ Department of Psychology, University of California, Berkeley, Berkeley CA, 94704

\begin{abstract}
David M. Sobel (dave sobel@brown.edu) James L. Morgan (james morgan@brown.edu)
Department of Cognitive, Linguistic, \& Psychological Sciences, Brown University, Providence RI, 02912
\end{abstract}

\begin{abstract}
A nascent understanding of absent reference emerges around 12 months: provided with rich contextual support, infants look and point to the location of a displaced object. When can infants understand absent reference without contextual support? Using a procedure modified from Hendrickson and Sundara (2017), 13- and 16-montholds first listened to utterances containing familiar target words, while viewing a checkerboard. Then, two objects - a referent and a distractor (e.g., a cup and a shoe) - appeared on the screen. Only 16-month-olds demonstrated a reliable looking preference for the referents, suggesting that listening to the utterances activated their mental images of the referents. These results establish that at 16 months, infants comprehend reference to absent entities without any contextual support.
\end{abstract}

Keywords: absent reference; unanchored absent reference; word learning; reference comprehension

Adult speakers routinely use language to communicate about remote places, imaginary situations, or past events. Intuitive and effortless to most adults, such use of language requires that the speaker recognizes that words act as symbolic references to objects, ideas, or events, even when they are not perceptually available at the time of verbal exchange. It is easy to see that understanding word meanings and recognizing their referential nature is an essential step in language acquisition, as it enables one to benefit from language-mediated learning and acquire information about the world that cannot be observed directly ("daddy is at work"). This raises the question of when and how infants gain the ability to recognize that words signify their referents, even when such referents are not in view.

Infants begin to show the first signs of recognizing the meanings of familiar words around 6-9 months (e.g., Benedict, 1979; Bergelson \& Swingley, 2012; Jusczyk \& Hohne, 1997) and utter their first words around 12-15 months (e.g., Benedict, 1979; Nelson, 1973). However, it is not until 16-20 months that infants begin to resolve referential ambiguity in word learning scenarios by recruiting a wide variety of social and referential cues (e.g., Baldwin, 1993a; Mather \& Plunkett, 2011; Tomasello, Strossberg, \& Akhtar, 1996) and existing word knowledge (e.g., Ferguson, Graf, \& Waxman, 2014). For example, 19-month-olds employ such cues to lay down a representation of a novel word referent even if it is not visible to them (e.g., Baldwin, 1993b). By 18-22 months, infants are capable not only of attending to referential cues but of making mutual exclusivity judgments when determining the referent of a novel word (Halberda, 2003), updating mental representations of absent objects that are being referred to in speech (Ganea, Shutts, Spelke, \& DeLoache, 2007), and become sensitive to semantic priming (Arias-Trejo \& Plunkett, 2009; Delle Luche, Durrant, Floccia, \& Plunkett, 2014; Mani \& Plunkett, 2010; see Wojcik, 2018, for review). In contrast, 10-month-olds defer to visual salience and ignore referential cues, such as gaze direction, when the referent of a novel word is ambiguous (Pruden, Hirsh-Pasek, Golinkoff, and Hennon, 2006). Similarly,12-month-olds are sensitive to referential cues but fail to recruit them for word learning (Hollich et al., 2000). What enables such a remarkable transition in infants' vocabulary acquisition in the second year?

We argue that one of the key forces that facilitates this transition is the advancement in infants' understanding of word-referent links. While lexical acquisition might initially be grounded in associative learning (e.g., Smith \& Yu, 2013) and not necessarily require an understanding that words refer, by the middle of the second year, infants attend to social and referential cues that help them infer the intended referent of a novel word (Brown, 1958; Golinkoff, Mervis, \& Hirsh-Pasek, 1994). In the present study, we report novel findings that suggest that the first half of the second year is marked by a developing comprehension of unanchored absent reference (defined below). We also discuss the possibility that comprehending unanchored reference to absent entities constitutes a qualitative shift in infants' understanding of the word-referent link and enables them to use words as an instrument of learning about the world, including facts and events one does not experience directly.

We define unanchored absent reference as the ability to call on a mental representation of a referent category upon hearing its name and without contextual support. Contextual support are events, such as hiding an object, that serve as visual or audiovisual primes for a given word-referent link. Such events constitute anchored absent reference - reference to an absent entity via a present object or activity (see Saylor, 2004). Having formed a stable link between words and mental representations (Saylor, 2004; Waxman \& Gelman, 2009) allows one to speak and comprehend speech about objects or events, even when the referents are not perceptually available (e.g., Deacon, 1997; Hollich, Hirsh-Pasek, \& Golinkoff, 2000; Werner \& Kaplan, 1963). Such an understanding is necessary for a language learner to create and update representations of the world upon 
receiving lexical input (De Saussure, 1916). When and how do infants develop the ability to comprehend unanchored absent reference? Two lines of research provide critical insight into the course of this development.

One line of research explored the relation between infants' acquisition of lexical, conceptual, and syntactic knowledge and evaluated their understanding of the word-referent link. For example, Xu, Cote, and Baker (2005) showed that by 12 months, infants can use words to individuate objects (i.e., two different words stand for two different objects, whereas the same word uttered twice stands for only one object). In their study, the experimenter introduced infants to an opaque box, looked inside and either uttered two novel labels, once each, or one novel label twice. She then retrieved one object from the box and let the infant explore the box. Infants' search time was longer when then experimenter uttered two distinct labels. Although it is not clear that 12-month-olds' understanding of words extends beyond appreciating the correspondence between the number of word types and the number of unique referents, infants at this age appear to realize that such a correspondence holds even for referents that are out of view. Similarly, Waxman and Markow (1995) demonstrated that 12-month-olds perceive words as invitations to form categories: infants' categorization was facilitated by hearing the same label applied to multiple images of objects. This indicates that 12-month-olds extend the meaning of newly learned words beyond the context in which they were learned. Such a comprehension suggests that 12-month-olds begin to map words onto concepts - one of the key markers of a referential understanding of words (Golinkoff, Mervis, \& Hirsch-Pasek, 1994). By 14 months, infants move beyond categorizing objects upon hearing the same noun-they begin to map novel properties to novel adjectives (Booth \& Waxman, 2009). Similarly, by 14-16 months, but not before, infants map familiar verbs onto animated images of actions (Bergelson and Swingley, 2015). The ability to establish such a mapping suggests that at least by 16 months, infants link different aspects of their experience to different grammatical categories-a hallmark of a referential understanding of words. Taken together, these findings delineate the unfolding of infants' understanding of the word-referent link and show a rapid progression in the infants' appreciation of the referential nature of words in the first half of the second year.

A complementary line of work investigated the development of reference in preverbal infants, such as pointing behavior and comprehension of anchored absent reference. Potentially originating from prelinguistic communicative exchanges (see Carpenter, Nagell, Tomasello, Butterworth, \& Moore, 1998), reference eventually enters infants'spoken language. Empirical evidence obtained in this line of work suggests that a proto-understanding of reference to absent entities is present by 12 months of age: infants begin to appreciate the referential nature of pointing (Gliga \& Csibra, 2009) and point to cues associated with a recently hidden object (see Liszkowski, Carpenter, \& Tomasello, 2007, for review). At 14 months, infants begin to interpret verbal requests for displaced objects using social information, such as individuals' history of interacting with an object, for disambiguation (Saylor \& Ganea, 2007). They also comprehend absent reference to familiar objects provided with minimal visual priming (i.e., minimal anchoring; Hendrickson \& Sundara, 2017). By 16 months, infants exhibit robust comprehension of anchored absent reference: they recognize properties (such as color) of recently removed objects whose labels are mentioned in utterances (Saylor, 2004) and produce communicative behaviors towards the anchors (e.g., pointing to the panel that occludes the absent referent).

These findings illustrate that the development of infants' referential behavior and anchored absent reference comprehension are closely aligned with the development of their understanding of the word-referent link: the more infants progress toward a referential understanding of words, the more skilled they become in mapping words onto absent entities.

Taken together, the body of evidence from both lines of research demonstrates that the acquisition of unanchored absent reference is a gradual process. This process accelerates between 12 and 18 months (see Ganea \& Saylor, 2013, for review; see also Huttenlocher, 1974), allowing infants to engage in progressively more sophisticated behaviors. Critically, the question this literature leaves open is the point at which infants comprehend unanchored absent reference.

The current study addresses this gap in the literature and establishes a method for testing infants' ability to understand unanchored absent reference to familiar categories. To achieve these aims, we developed a procedure based on Hendrickson and Sundara (2017). In their experiment, infants began with a pretest, in which they viewed images of four objects in silence (which constitute the minimal priming mentioned above). Then, they listened to utterances containing labels for two of the previously displayed objects while looking at an unrelated image. Infants then viewed the original four objects again, and their preference for looking to the two previously labeled objects was measured in an infant-controlled procedure. Fourteen-month-olds were able to correctly identify the referents of labels they had heard in the utterances, looking longer to these referents during the post-test.

Because Hendrickson and Sundara's procedure involved pretest exposure to the images used at test, it is possible that infants' looking behavior on test trials was influenced, at least in part, by a priming effect - infants may have (1) retained the perceptual properties of the images through the audio exposure and (2) expected that the recently shown images would be the topic of the subsequent utterances. To avoid such anchoring, we modified the procedure by removing the pretest phase. We also limited the theme of the utterances to one referent to enable the use of an experimenter-controlled preferential looking procedure. Thus, on test trials, infants saw two objects, and the proportion of their looking to the previously referenced object (the target) was measured. Our goal was to see whether infants at 13 and 16 months can demonstrate an understanding of unanchored absent reference. If they do have such an understanding, they should look longer to the target referent. 


\section{Method}

\section{Participants}

Forty-one infants ( $M$ age $=450$ days, $S D=48.29 ; 20$ girls, 21 boys) were recruited using state birth records and Facebook ads from Providence, RI, and Berkeley, CA, areas. Twenty-two additional participants were tested but excluded from analyses due to fussiness $(N=14)$ and eyetracker calibration failure $(N=8)$. All participants were monolingual learners of American English (less than 10\% of exposure to other languages at home or daycare) with no recent ear infections or history of diagnosed developmental disabilities. Each participant received a toy, a t-shirt, or a book. We did not collect data on participants' SES, race, or ethnicity. Most participants came from lower- to uppermiddle class families and were Caucasian.

Because we aimed to establish the age at which infants begin to comprehend references to absent entities without contextual support, we split our dataset into two halves by age. Earlier investigations of infants' understanding of anchored absent reference reported a significant difference between 13- and 16month-olds (Ganea \& Saylor, 2013a). Unlike the younger infants, 16-month-olds were able to comprehend reference to a recently introduced absent person. In a different study, 15month-olds responded to a reference to an absent person, whereas 12-month-olds showed no discernible response (Saylor \& Baldwin, 2004).

Based on these findings, we predicted that the older half of our sample $\left(N=20 ; M_{\text {age }}=16.11\right.$ months, $S D_{\text {age }}=0.97$ months, 11 girls) would be more likely to exhibit comprehension of unanchored reference to absent objects than the younger half $\left(N=21 ; M_{\text {age }}=13.43\right.$ months, $S D_{\text {age }}=0.68$ months; 9 girls $)$.

\section{Apparatus}

Infants sat on a caretaker's lap for the entire duration of the procedure. On both testing sites, stimuli were presented on a screen (Dell 19" flat screen monitor) mounted on a wall approximately $90 \mathrm{~cm}$ in front of the seat. Audio stimuli were presented via a Sentry $110 \mathrm{~A}$ monitor speaker that played the auditory stimuli at a conversational level (75 dB). We used an SMI red 250 remote binocular eye-tracker to record participants' looking behavior.

\section{Materials}

Audio stimuli The utterances containing the target familiar labels (nouns: ball, shoe, cup, tree) were recorded by a female native speaker of American English from Rhode Island. All recordings were produced in a sound-proof booth and edited using Praat (Boersma \& Weenink, 2005).

Visual stimuli The images of objects used on test trials were .png-formatted photographs of the objects corresponding to the labels mentioned in utterances. Six adult native speakers of American English were recruited to rate (from 1 "not at all a good example" to 5 "an excellent example") a set of candidate images as typical exemplars of the four target categories. The highest-rated images that represented a toy ball, a shoe, a sippy cup, and a tree were chosen as experimental stimuli (Figure 1).
We asked caregivers to complete a survey about their infants' receptive and productive vocabulary and motor competence. We also asked caregivers to report infant's exposure to screen media in a typical week.

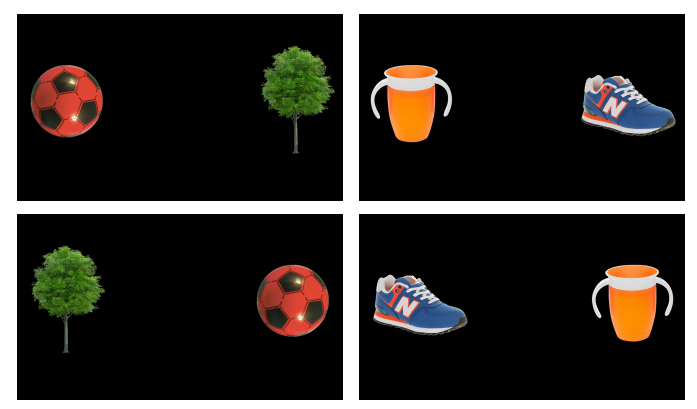

Figure 1. Images used as visual stimuli

\section{Design and Procedure}

The researcher brought the infant and the caregiver to the dimly lit room. The infant sat on the caregiver's lap in front of a computer screen with a binocular eye-tracker mounted underneath it. The caregiver was asked to wear opaque sunglasses during the procedure to ensure that only the infants' eyes were registered by the eye-tracker. Then the eye-tracking calibration began. The infant saw an image of a kitten in four corners of the screen and at its center and heard "meow" noises. Calibration was repeated until it was successful.

After calibration, the experimental procedure began. Each trial consisted of an auditory exposure phase and a test phase. During the auditory exposure phase, infants heard a 6-sentence utterance containing one of the target referents (ball, shoe, tree, or cup; e.g., "I have a ball. My ball is round. I kick my ball in my yard. My ball can bounce. I put my ball on the floor. I will show you my ball.") and saw a static checkerboard on the screen. The image was displayed to help sustain infants' attention on the auditory stimuli and was unrelated to the meaning of phrases in the utterances. After the last word was uttered, infants saw a black screen for 1 second before the test phase began. We did not elicit central fixation before the test trials because we analyzed the differences in infants' looking to one object of a pair (so that any looking bias is cancelled out) depending on whether it was a target or a distractor. During test, infants saw two objects presented on the right and the left of the screen, against a dark background. The images were presented in silence and remained on the screen for 5.5 seconds. This trial duration was chosen based on earlier work that employed the preferential looking procedure with infants of similar ages (e.g., Ferguson et al., 2014).

Because there were four images corresponding to the four words from the utterances, each image was used once as the target and once as the distractor (ball was paired with tree and shoe was paired with cup; see Figure 1), such that a baseline preference could be averaged away for the purpose of the analyses. To minimize the possibility that infants' looking behavior on trials 3 and 4 was influenced by a potential priming effect (i.e., seeing the same images on trials 1 and 2), the order of trial presentation was counterbalanced between participants, 
who were randomly assigned to one of two presentation orders (either ball-shoe-tree-cup, or tree-cup-ball-shoe).

\section{Data Coding and Analyses}

To assess infants' looking behavior, we aggregated the data into a series of 500-ms bins. The dependent variable on test trials was the amount of looking time to the target object divided by the total looking time to the target and the distractor, calculated for each 500-ms bin in the 5.5-second window.

For each trial, two rectangular areas of interest were defined around each of the images. Gazes outside these areas were excluded from analysis. When the eye-tracking data were missing for a given bin but recorded for the bins before and after, we replaced the missing data by calculating the average proportion of looking time for the two neighboring bins. In cases when two or more bins had missing data, no replacement was conducted. To minimize potential priming effects or order effects, we calculated the difference in looking behavior to the one object for each pair (ball for the ball-tree pair and shoe for the shoe-cup pair) on trials when that object was the target and trials when it was the distractor (i.e., [\% of LT to the ball when ball was target] minus [\% of LT to ball when tree was target]).

\section{Results}

In general, infants were engaged with the task and looked to the screen $80 \%$ of the time during the test phase: while many infants looked away from the screen during audio exposure, the majority looked to the screen when the images appeared at test. Figure 2a shows the average difference in the proportion of infants' looking to the target during the 5.5-second window of analysis. Figure $2 b$ shows the difference in the proportion of their looking to the target object during each time bin.

Before conducting the planned analyses, we ran ShapiroWilk normality tests on the 5.5 -second average difference in the proportion of looking time (hereafter, "aggregate looking preference score"). This was done to ensure that the identity link function was appropriate for the generalized linear mixed model (GLMM). The test revealed that the distribution, albeit underlyingly bounded within the $[-1,1]$ interval, did not significantly deviate from normal, $p=0.12$. We conducted subsequent analyses using conventional parametric assumptions and used the identity link function in the GLMM.

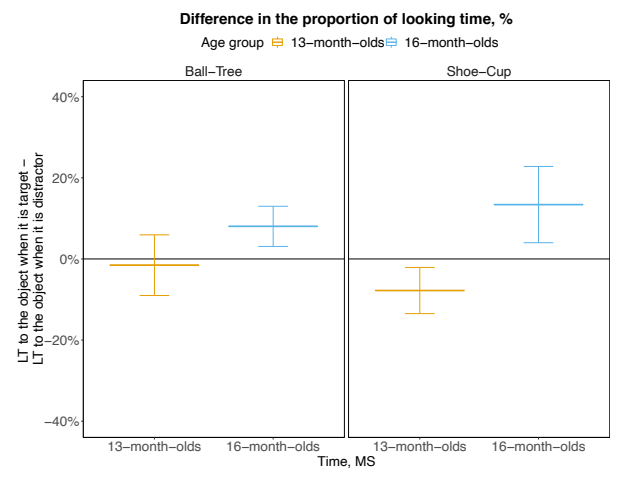

Figure 2a. Average differences in the proportion of infants' looking time

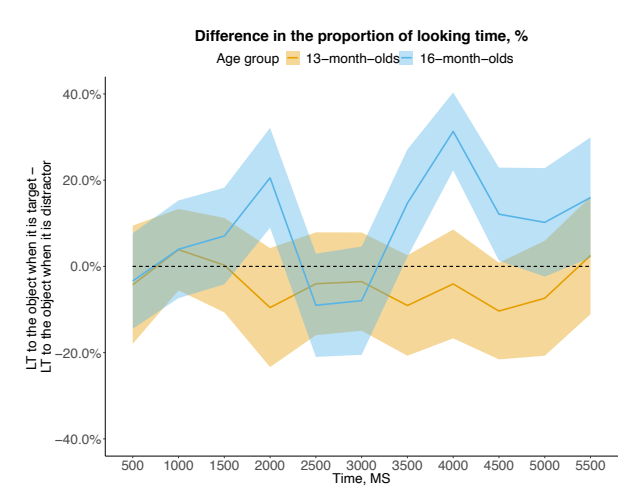

Figure 2b. Difference in the proportion of infants' looking time for each time bin

We ran preliminary tests to see whether infants' vocabulary level, motor competence, and exposure with screen media had significant effects on their looking behavior. The measures of screen exposure were included in order to evaluate whether the novelty of experience (seeing 2D representations of objects on the screen and hearing a digital recording of a voice) contributed to the differences in infants' looking behavior. The preliminary analyses were conducted by fitting a GLMM to the aggregate looking preference score. Infant's receptive and productive vocabulary, gender, motor competence, infant's exposure to screen media, and the order of stimuli presentation were entered as predictors. No predictor had a significant effect on the difference in infants' looking time, all $p$-values $>.08$. These variables were excluded from subsequent analyses.

To evaluate infants' looking behavior and test our hypothesis, we fitted a GLMM to the aggregate looking preference score and compared the differences for each bin using one-tailed t-tests. We used one-tailed tests because we predicted that older infants will be more likely to show a larger difference in looking time than younger infants. For the GLMM, we evaluated the fixed effect of age group (13-montholds and 16-month-olds) and the random effect of image pair, while enforcing a zero correlation between the intercept deviations and the effect deviations of age group across image pairs. The reason for including image pair as a random factor was to account for the effects of infants' receptive vocabulary: according to parental surveys only 7 out of 41 infants were familiar with the word "tree", whose referent was paired with an image of a ball on test trials (see Table 1). We also evaluated the difference in looking time against chance (i.e. equivalent looking time to the image when it was the target and when it was the distractor, so that the aggregate looking preference score is 0 ) for each age group using two-tailed t-tests.

Table 1. Infants' knowledge of the target words

\begin{tabular}{lcc}
\hline Word & 13-month-olds & 16-month-olds \\
\hline Ball & 13 & 16 \\
Shoe & 13 & 16 \\
Tree & 2 & 5 \\
Cup & 8 & 16 \\
\hline
\end{tabular}


Consistent with our predictions, the analyses revealed a significant effect of age group, $t=2.21, p=0.03$, with 16 month-olds demonstrating a larger difference in looking time than 13-month-olds. The image pair did not produce a significant effect on infants' looking behavior. Figure 2a demonstrates similar patterns of difference in looking time for 13- and 16-month-olds for both image pairs. One-tailed t-tests revealed a significant divergence between the groups at 2000 $\mathrm{ms}, t=1.67, p=.05$, and $4000 \mathrm{~ms}, t=2.28, p=.01$, and a nearly significant difference at $3500 \mathrm{~ms}, p=.09$ (see Figure $2 b)$. The comparison of the aggregate looking preference score for each age group with chance, revealed that only 16-montholds had a reliable preference for target images, $t=2.09, p=$ .04 . Younger infants looked equivalently when a given image was the target and the distractor, $p=.31$ (see Figure 2a). In addition to running our planned analyses with two age groups, we fitted an additional GLMM to the difference in looking time with infants' age in days as a continuous variable. This was done to see whether the gradual progression observed in infants' growing appreciation of the referential nature of words and their increasing ability to comprehend anchored absent reference holds for their unanchored absent reference comprehension. Expectedly, the effect of age was significant, $t=2.46, p=0.02$.

The results of our analyses indicate that at least by 16 months, infants are significantly more likely to look to the object mentioned in an utterance than to an unrelated familiar object. Consistent with our predictions, this finding suggests that 16-month-olds activate mental representations of familiar objects upon just hearing their names embedded in simple utterances. Unlike the younger infants in our sample, the 16month-olds could comprehend unanchored absent reference. Curiously, the observed pattern of significant divergences in infants' looking behavior during test is consistent with the pattern found in LaTourrette and Waxman, (2019): in the absence of auditory stimuli at test, the divergence in infants' looking preference emerges around 3500-3800 ms after the onset of visual stimuli.

\section{Discussion}

Our findings establish that at 16 months infants begin to comprehend unanchored absent reference. Infants activate mental representations of objects upon hearing their names embedded in utterances without having recently seen or interacted with those objects. This ability appears to emerge between 13 and 16 months, mirroring the development of infants' growing appreciation of the referential nature of words and their overall ability to comprehend anchored reference to absent entities. Before 16 months, one can observe hints of this ability, but no explicit evidence of unanchored absent reference has been obtained for younger infants. Younger infants' sensitivity to visual or multimodal priming (i.e., anchoring), which enables them to understand anchored reference to recently displaced objects (Ganea \& Saylor, 2013a), suggests that the capacity for absent reference comprehension is in place earlier than 16 months. While 13-month-olds require extensive contextual support (i.e., anchors) to comprehend reference to absent entities, 14- to 15-month-olds are capable of such comprehension after minimal visual priming (Ferguson et al., 2014; Hendrickson \& Sundara, 2017). The present findings show that at 16 months, infants can understand unprimed, or unanchored, references to absent familiar objects.

The developmental trajectory of infants' comprehension of unanchored absent reference suggested by our findings is consistent with the broader literature on infants' use of referential and social cues in word learning and comprehension. By 16 months, it undergoes a significant shift, enabling infants to engage in increasingly sophisticated forms of word learning. For example, by 16 months, infants become sensitive to false labeling events (while taking into account speakers' access to information) and attempt to interrupt and correct erroneous statements (Koenig \& Echols, 2003). At 18 months, they are able to learn novel words selectively based on speakers' history of false labeling (Luchkina, Sobel, \& Morgan, 2018). Eighteen-month-olds also begin to recognize the relation between pointing and labeling, and they maintain attention on labeled objects (Baldwin \& Markman, 1989).

In sum, the accumulated body of evidence, including the present findings, suggests that the ability to comprehend unanchored reference develops gradually between 12 and 16 months of age and constitutes a significant milestone in infants' language acquisition. Having accomplished such a comprehension enables a qualitative shift in infants' ability to learn from decontextualized speech and likely facilitates further development of their representational abilities. The present work establishes for the first time that by 16 months, infants comprehend verbal reference to entities that are not perceptually available.

A limitation of our findings is that infants' knowledge of the words used during test correlated with age, $p<0.01$. Thus, there remains a possibility that the comprehension of unanchored absent reference is present at earlier ages. Additionally, while earlier research, combined with the present findings, suggests that infants' performance on test trials was driven by their calling on mental representations of categories corresponding to the target words, there is a chance that word forms remained active into the test phase and drove infants' looking behavior via associative mechanisms. Although our experimental design cannot rule out this possibility, it would be surprising if the word form stayed active for the $5.5 \mathrm{~s}$ duration of the trial: ERP correlates of word-form activation are typically present within $1000 \mathrm{~ms}$ from the word onset (e.g. Becker, Schild, \& Friedrich, 2014).

A follow-up investigation that examines when and how infants gain the ability to learn novel words that describe absent referents will help address the remaining concerns about the mechanisms underlying infants' looking behavior in the present work. If infants can learn the meanings of novel words without seeing their referents and subsequently draw on that knowledge to select the referents of those words, it will constitute strong evidence that infants' word knowledge at 16 months entails reference and cannot be reduced to associative learning. 


\section{References}

Arias-Trejo, N., \& Plunkett, K. (2009). Lexical-semantic priming effects during infancy. Philosophical Transactions of the Royal Society B: Biological Sciences, 364(1536), 3633-3647.

https://doi.org/10.1098/rstb.2009.0146

Baldwin, D. A. (1993a). Early referential understanding: Infants' ability to recognize referential acts for what they are. Developmental Psychology, 29(5), 832-843. https://doi.org/10.1037/0012-1649.29.5.832

Baldwin, D. A. (1993b). Infants' ability to consult the speaker for clues to word reference. Journal of Child Language, 20(2), 395-418. https://doi.org/10.1017/S0305000900008345

Baldwin, D. A., \& Markman, E. M. (1989). Establishing Word-Object Relations: A First Step. Child Development, 60(2), 381. https://doi.org/10.2307/1130984

Becker, A. B. C., Schild, U., \& Friedrich, C. K. (2014). ERP correlates of word onset priming in infants and young children. Developmental Cognitive Neuroscience, 9, 4455. https://doi.org/10.1016/j.dcn.2013.12.004

Benedict, H. (1979). Early lexical development: Comprehension and production. Journal of Child Language, 6(2), 183-200. https://doi.org/10.1017/S0305000900002245

Bergelson, E., \& Swingley, D. (2012). At 6-9 months, human infants know the meanings of many common nouns. Proceedings of the National Academy of Sciences, 109(9), 3253-3258.

https://doi.org/10.1073/pnas.1113380109

Bergelson, Elika, \& Swingley, D. (2015). Early Word Comprehension in Infants: Replication and Extension. Language Learning and Development, 11(4), 369-380. https://doi.org/10.1080/15475441.2014.979387

Boersma, P., \& Weenink, D. (2005). Doing phonetics by computer (Version 5.1).

Booth, A. E., \& Waxman, S. R. (2009). A Horse of a Different Color: Specifying With Precision Infants' Mappings of Novel Nouns and Adjectives. Child Development, 80(1), 15-22. https://doi.org/10.1111/j.1467-8624.2008.01242.x

Brown, R. (1958). Words and things. Free Press.

Carpenter, M., Nagell, K., Tomasello, M., Butterworth, G., \& Moore, C. (1998). Social Cognition, Joint Attention, and Communicative Competence from 9 to 15 Months of Age. Monographs of the Society for Research in Child Development, 63(4), i. https://doi.org/10.2307/1166214

De Saussure, F. (1916). Course in general linguistics, ed. Charles Bally and Albert Sechehaye. La Salle, IL: Open Court Publishing.

Deacon, T. (1997). The symbolic species. New York: W. Delle Luche, C., Durrant, S., Floccia, C., \& Plunkett, K. (2014). Implicit meaning in 18-month-old toddlers. Developmental Science, 17(6), 948-955. https://doi.org/10.1111/desc. 12164

Ferguson, B., Graf, E., \& Waxman, S. R. (2014). Infants use known verbs to learn novel nouns: Evidence from 15- and 19-month-olds. Cognition, 131(1), 139-146. https://doi.org/10.1016/j.cognition.2013.12.014

Ganea, P. A., \& Saylor, M. M. (2013a). Talking about the near and dear: Infants' comprehension of displaced speech. Developmental Psychology, 49(7), 1299-1307. https://doi.org/10.1037/a0030086

Ganea, P. A., \& Saylor, M. M. (2013b). Representational Constraints on Language Development: Thinking and Learning About Absent Things. Child Development Perspectives, 7(4), 227-231. https://doi.org/10.1111/cdep.12045

Ganea, P. A., Shutts, K., Spelke, E. S., \& DeLoache, J. S. (2007). Thinking of Things Unseen: Infants' Use of Language to Update Mental Representations. Psychological Science, 18(8), 734-739.

Gliga, T., \& Csibra, G. (2009). One-year-old infants appreciate the referential nature of deictic gestures and words. Psychological Science, 20(3), 347-353. https://doi.org/10.1111/j.1467-9280.2009.02295.x

Golinkoff, R. M., Mervis, C. B., \& Hirsh-Pasek, K. (1994). Early object labels: The case for a developmental lexical principles framework. Journal of Child Language, 21(1), 125-155. https://doi.org/10.1017/S0305000900008692

Halberda, J. (2003). The development of a word-learning strategy. Cognition, 87(1), B23-B34. https://doi.org/10.1016/S0010-0277(02)00186-5

Hendrickson, K., \& Sundara, M. (2017). Fourteen-montholds' decontextualized understanding of words for absent objects. Journal of Child Language, 44(1), 239-254. https://doi.org/10.1017/S0305000915000756

Hollich, G., Hirsh-Pasek, K., \& Golinkoff, R. M. (2000). I. What Does it Take to Learn a Word? Monographs of the Society for Research in Child Development, 65(3), 1-16. https://doi.org/10.1111/1540-5834.00091

Hollich, G. J., Hirsh-Pasek, K., Michnick Golinkoff, R., Brand, R. J., Brown, E., Chung, H. L., Hennon, E., Rocroi, C., \& Bloom, L. (2000). Breaking the Language Barrier: An Emergentist Coalition Model for the Origins of Word Learning. In Monographs of the Society for Research in Child Development, Vol. 65, No. 3 (pp. ivi+1-135). Wiley on behalf of the Society for Research in Child Development. https://www.jstor.org/stable/3181533

Huttenlocher, J. (1974). The origins of language comprehension. In Theories in cognitive psychology: The Loyola Symposium. Lawrence Erlbaum.

Jusczyk, P. W., \& Hohne, E. A. (1997). Infants' Memory for Spoken Words. Science, 277(5334), 1984-1986. https://doi.org/10.1126/science.277.5334.1984

Koenig, M. A., \& Echols, C. H. (2003). Infants' understanding of false labeling events: The referential roles of words and the speakers who use them. Cognition, 87(3), 179-208. https://doi.org/10.1016/S00100277(03)00002-7

LaTourrette, A., \& Waxman, S. R. (2019). A little labeling goes a long way: Semi-supervised learning in infancy. Developmental Science, 22(1), e12736. https://doi.org/10.1111/desc.12736 
Liszkowski, U., Carpenter, M., \& Tomasello, M. (2007). Pointing out new news, old news, and absent referents at 12 months of age. Developmental Science, 10(2), F1-F7. https://doi.org/10.1111/j.1467-7687.2006.00552.x

Luchkina, E., Sobel, D. M., \& Morgan, J. L. (2018). Eighteen-month-olds selectively generalize words from accurate speakers to novel contexts. Developmental Science, 21(6), e12663. https://doi.org/10.1111/desc. 12663

Mani, N., \& Plunkett, K. (2010). In the Infant's Mind's Ear: Evidence for Implicit Naming in 18-Month-Olds.

Psychological Science, 21(7), 908-913. https://doi.org/10.1177/0956797610373371

Mather, E., \& Plunkett, K. (2011). Mutual exclusivity and phonological novelty constrain word learning at 16 months. Journal of Child Language, 38(5), 933-950. https://doi.org/10.1017/S0305000910000401

Nelson, K. (1973). Structure and Strategy in Learning to Talk. Monographs of the Society for Research in Child Development, 38(1/2), 1. https://doi.org/10.2307/1165788

Pruden, S. M., Hirsh-Pasek, K., Golinkoff, R. M., \& Hennon, E. A. (2006). The Birth of Words: Ten-MonthOlds Learn Words Through Perceptual Salience. Child Development, 77(2), 266-280. https://doi.org/10.1111/j.1467-8624.2006.00869.x

Saylor, M. M. (2004). Twelve- and 16-month-old infants recognize properties of mentioned absent things. Developmental Science, 7(5), 599-611. https://doi.org/10.1111/j.1467-7687.2004.00383.x

Saylor, M. M., \& Baldwin, D. A. (2004). Discussing those not present: Comprehension of references to absent caregivers. Journal of Child Language, 31(3), 537-560. https://doi.org/10.1017/S0305000904006282

Saylor, M. M., \& Ganea, P. (2007). Infants interpret ambiguous requests for absent objects. Developmental Psychology, 43(3), 696-704. https://doi.org/10.1037/0012-1649.43.3.696

Smith, L. B., \& Yu, C. (2013). Visual Attention Is Not Enough: Individual Differences in Statistical WordReferent Learning in Infants. Language Learning and Development, 9(1), 25-49. https://doi.org/10.1080/15475441.2012.707104

Tomasello, M., Strosberg, R., \& Akhtar, N. (1996). Eighteen-month-old children learn words in non-ostensive contexts. Journal of Child Language, 23(1), 157-176. https://doi.org/10.1017/S0305000900010138

Waxman, S. R., \& Gelman, S. A. (2009). Early wordlearning entails reference, not merely associations. Trends in Cognitive Sciences, 13(6), 258-263. https://doi.org/10.1016/j.tics.2009.03.006

Waxman, S. R., \& Markow, D. B. (1995). Words as Invitations to Form Categories: Evidence from 12- to 13Month-Old Infants. Cognitive Psychology, 29(3), 257302. https://doi.org/10.1006/cogp.1995.1016

Werner, H., \& Kaplan, B. (1963). Symbol formation. An organismic - developmental approach to language and expression of thought. New York: John Wiley \& Sons, Inc.

Wojcik, E. H. (2018). The Development of LexicalSemantic Networks in Infants and Toddlers. Child Development Perspectives, 12(1), 34-38. https://doi.org/10.1111/cdep.12252

Xu, F., Cote, M., \& Baker, A. (2005). Labeling guides object individuation in 12-month-old infants. Psychological Science, 16(5), 372-377. https://doi.org/10.1111/j.0956-7976.2005.01543.x 